The Astrophysical Journal, 681:626-635, 2008 July 1

(C) 2008. The American Astronomical Society. All rights reserved. Printed in U.S.A.

\title{
A RESOLVED MOLECULAR GAS DISK AROUND THE NEARBY A STAR 49 CETI
}

\author{
A. M. Hughes, ${ }^{1}$ D. J. Wilner, ${ }^{1}$ I. Kamp, ${ }^{2}$ And M. R. Hogerheidde ${ }^{3}$ \\ Received 2008 January 8; accepted 2008 March 20
}

\begin{abstract}
The A star 49 Ceti, at a distance of $61 \mathrm{pc}$, is unusual in retaining a substantial quantity of molecular gas while exhibiting dust properties similar to those of a debris disk. We present resolved observations of the disk around 49 Ceti from the Submillimeter Array in the $J=2-1$ rotational transition of CO with a resolution of $1.0^{\prime \prime} \times 1.2^{\prime \prime}$. The observed emission reveals an extended rotating structure viewed approximately edge-on and clear of detectable $\mathrm{CO}$ emission out to a distance of $\sim 90$ AU from the star. No $1.3 \mathrm{~mm}$ continuum emission is detected at a $3 \sigma$ sensitivity of $2.1 \mathrm{mJy} \mathrm{beam}^{-1}$. Models of disk structure and chemistry indicate that the inner disk is devoid of molecular gas, while the outer gas disk between 40 and $200 \mathrm{AU}$ from the star is dominated by photochemistry from stellar and interstellar radiation. We determine parameters for a model that reproduces the basic features of the spatially resolved CO $J=2-1$ emission, the spectral energy distribution, and the unresolved CO $J=3-2$ spectrum. We investigate variations in disk chemistry and observable properties for a range of structural parameters. The star 49 Ceti appears to be a rare example of a system in a late stage of transition between a gas-rich protoplanetary disk and a tenuous, virtually gasfree debris disk.
\end{abstract}

Subject headings: astrochemistry — circumstellar matter — planetary systems: protoplanetary disks — stars: individual (49 Ceti)

\section{INTRODUCTION}

A key to understanding the formation of planetary systems lies in characterizing the transitional phase between the gas-rich primordial disks found around young $\mathrm{T}$ Tauri stars and the tenuous, virtually gas-free debris disks around their main-sequence counterparts. Unfortunately, disks in this transitional phase are rare and difficult to identify. Dust disks around young stars are commonly identified through the "Vega-excess" phenomenon (first observed using the Infrared Astronomical Satellite [IRAS] by Aumann et al. 1984; see review by Zuckerman 2001), in which an infrared excess over the stellar photosphere is attributed to reprocessing of optical and ultraviolet starlight by thermally emitting circumstellar dust grains. The star 49 Ceti was first identified in this way by Sadakane \& Nishida (1986). The quantity $\tau=L_{\mathrm{IR}} / L_{\mathrm{bol}}$ is often used as an indicator of the "optical depth" of the dust disk, as it provides a rough estimate of the quantity of optical/ ultraviolet light intercepted and reemitted by the dust. Jura et al. (1993) correlated the IRAS Point Source Catalog with the Yale Bright Star Catalog (Hoffleit \& Jaschek 1991) and identified three A stars with $\tau>10^{-3}$, indicative of tenuous, optically thin circumstellar dust. Two were the stars $\beta$ Pic and HR 4796, which are now known to host debris disks. The third was 49 Ceti, which unlike the other two defies classification as a debris disk because it retains a substantial quantity of molecular gas, first observed in the $\mathrm{CO} J=2-1$ line (Zuckerman et al. 1995) and later confirmed in $J=3-2$ (Dent et al. 2005). At a distance of only 61 pc (Hipparcos), it is one of the closest known gas-rich circumstellar disks, farther only than TW Hydrae (51 pc; Mamajek 2005). Its outward similarity to a debris disk, combined with the substantial quantity of molecular gas still present in the system, suggest that the disk may be in an unusual transitional evolutionary phase.

${ }^{1}$ Harvard-Smithsonian Center for Astrophysics, 60 Garden Street, Cambridge, MA 02138

2 Kapteyn Astronomical Institute, University of Groningen, 9700 AV Groningen, Netherlands.

${ }^{3}$ Leiden Observatory, Leiden University, P.O. Box 9513, 2300 RA Leiden, Netherlands.
All three high- $\tau$ A stars are young: HR 4796A has an age of $8 \pm 2$ Myr (Stauffer et al. 1995), and $\beta$ Pic has been placed at $\sim 12$ Myr (Zuckerman et al. 2001; Ortega et al. 2002). The age of 49 Ceti is uncertain due to its isolation; unlike $\beta$ Pic or HR 4796A there are no known associated low-mass stars to provide a corroborating age estimate. Jura et al. (1998) demonstrate that on a Hertzsprung-Russell (H-R) diagram, all three stars exhibit a low luminosity for their color, which is likely attributable to their young ages $(\sim 10 \mathrm{Myr})$. Using the evolutionary tracks of Siess et al. (2000), Thi et al. (2001b) assign an age of 8 Myr to 49 Ceti based on its position on the H-R diagram.

Few conclusive measurements have been made of the dust properties in the 49 Ceti system. HST NICMOS coronagraphic observations of 49 Ceti failed to detect any scattered light in the near-infrared at $r>1.6^{\prime \prime}$ (Weinberger et al. 1999). Recent subarcsecond-scale imaging at mid-infrared wavelengths with Keck (Wahhaj et al. 2007) revealed dust emission at 12.5 and $17.9 \mu \mathrm{m}$, extended along a northwest-southeast axis and apparently inclined at an angle of $60^{\circ}$. Simple models of the dust emission suggest a radial size segregation of dust grains, with a population of very small grains $(a \sim 0.1 \mu \mathrm{m})$ confined between 30 and $60 \mathrm{AU}$ from the star and a population of larger grains $(a \sim 15 \mu \mathrm{m})$ from 60 to 900 AU from the star. However, the outer radius of this latter component is uncertain due to its dependence on the millimeter flux, which is not well determined. There are two contradictory single-dish measurements of the millimeter dust emission, both with modest signal-to-noise ratio $(\mathrm{S} / \mathrm{N})$. Bockelée-Morvan et al. (1994) report a IRAM 1.2 mm flux of $12.7 \pm 2.3 \mathrm{mJy}$, while Song et al. (2004) report a JCMT SCUBA $850 \mu \mathrm{m}$ flux of $8.2 \pm 1.9 \mathrm{mJy}$. These measurements are mutually inconsistent for either a thermal spectrum $\left(F_{\lambda} \propto \lambda^{-2}\right)$ or a typical optically thin circumstellar disk spectrum $\left(F_{\lambda} \propto \lambda^{-3}\right)$ in this wavelength regime.

If we accept the lower value of the $850 \mu \mathrm{m}$ flux and make standard assumptions about the dust opacity (e.g., Beckwith \& Sargent 1991), then the total mass of the 49 Ceti dust disk is $0.1 M_{\oplus}$. If we compare this to other nearby dusty disks at potentially similar stages of evolution, we find that 49 Ceti, with an $850 \mu \mathrm{m}$ flux of 
$8.2 \mathrm{mJy}$ at a distance of $61 \mathrm{pc}$, has a dust mass $\left(\propto F_{850 \mu \mathrm{m}} d^{2}\right)$ approximately $80 \%$ that of $\beta$ Pic (104.3 mJy, $19.3 \mathrm{pc}$; Holland et al. 1998) but only $0.3 \%$ that of the typical Herbig Ae star HD 169142 (554 mJy, 145 pc; Sylvester et al. 1996). Thus, the 49 Ceti disk appears to have a tenuous dust disk more akin to that of the debris disk around $\beta$ Pic than a gas-rich protoplanetary disk.

Studies of the distribution of gas in the 49 Ceti system have been similarly inconclusive, particularly since it is not obvious that a substantial reservoir of molecular gas should persist in the strong UV field of an A star at this apparently advanced stage. Attempts to detect pure rotational transitions of the $\mathrm{H}_{2}$ molecule have resulted in contradictory reports, with Thi et al. (2001a) reporting a marginal detection using SWS ISO, which Chen et al. (2006) did not confirm with Spitzer IRS observations; nor did Carmona et al. (2008) detect $\mathrm{H}_{2}$ emission with VLT CRIRES observations. Models of the double-peaked JCMT CO $J=3-2$ line profile observed by Dent et al. (2005) indicated that the gas is likely distributed in either a very compact disk with $\sim 16^{\circ}$ inclination or a more inclined ring of radius $\sim 50$ AU and inclination $\sim 35^{\circ}$. The latter was deemed more consistent with the dust distribution seen in the mid-infrared, although it fails to reproduce the high-velocity wings that may be present in the $\mathrm{CO}$ $J=3-2$ line profile.

In order to obtain spatially resolved information on the distribution of material in the system, we observed 49 Ceti with the Submillimeter Array (SMA) in the $J=2-1$ transition of CO and associated continuum. ${ }^{4}$ We detect a rotating structure of much greater extent than predicted from the single-dish measurements, with a large central region devoid of molecular gas emission. We also model the disk emission using COSTAR (Kamp \& Bertoldi 2000; Kamp \& van Zadelhoff 2001), a code that combines thin hydrostatic equilibrium models of disks with a rich chemistry network and a detailed heating and cooling balance to determine gas properties. The models provide some insight into basic properties of the disk, including the region of photodissociation of $\mathrm{CO}$ in the inner disk and the spatial extent of the emission.

The observations are described in $\S 2$, and results are presented in $\S 3$. In $\S 4$ we discuss the process undertaken to model the data, including the basic model structure, the initial conditions for the chemistry, and the initial model adopted from the dust emission analysis of Wahhaj et al. (2007), as well as adjustments to that fiducial model necessitated by the new observations. The parameter space is explored in $\S 4.3$, including an investigation of the varying influence of chemistry across the model grid, and $\S 4.4$ discusses the dust properties in the context of the spectral energy distribution (SED) predicted from the gas model. The best-fit model is discussed in $\S 4.5$, including an a posteriori comparison of the model prediction with the observed CO $J=3-2$ spectrum; inadequacies of the model are also noted. The results are discussed in the broader context of disk evolution in $\S 5$, and a summary is presented in $\S 6$.

\section{OBSERVATIONS}

We observed 49 Ceti with the SMA at $230 \mathrm{GHz}$ during an $11 \mathrm{hr}$ track on the night of 2006 October 13. Atmospheric phase was extremely stable, with typical phase changes of $<15^{\circ}$ between calibrator scans (every 25 minutes). Seven antennas were used in the "extended" configuration, with projected baselines between 15 and $130 \mathrm{~m}$. The primary flux calibrator was Uranus,

\footnotetext{
4 The Submillimeter Array is a joint project between the Smithsonian Astrophysical Observatory and the Academia Sinica Institute of Astronomy and Astrophysics and is funded by the Smithsonian Institution and the Academia Sinica.
}

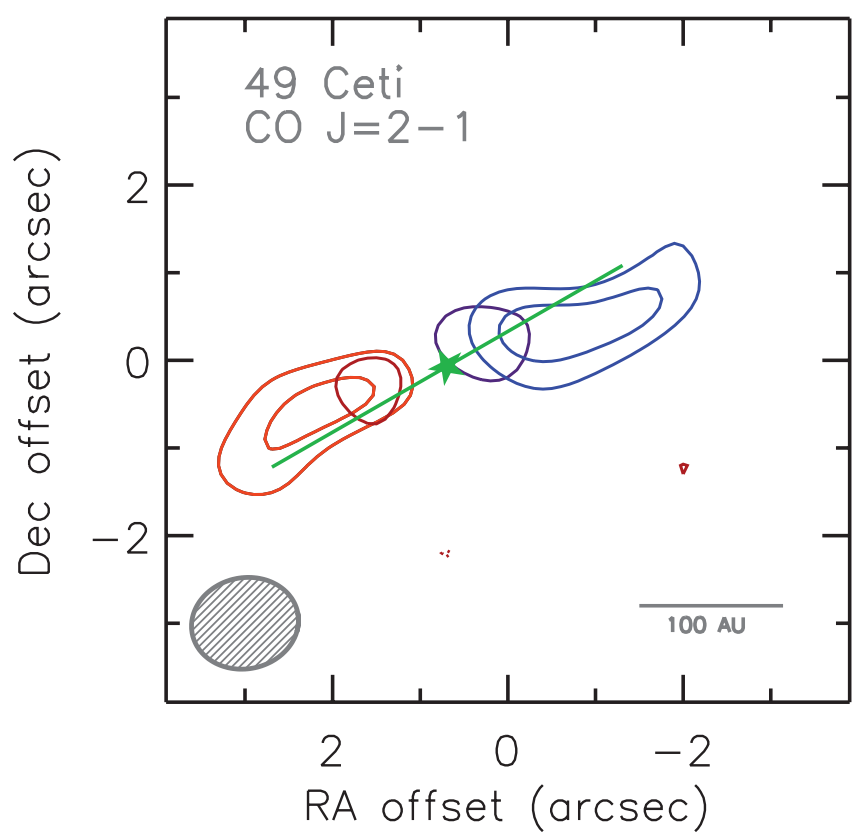

FIG. 1.- Renzogram of SMA observations of 49 Ceti in the CO $J=2-1$ line. The beam size is $1.0^{\prime \prime} \times 1.2^{\prime \prime}$, and the position angle is $-79^{\circ}$. Contours are $-3 \times 37,3 \times 37$, and $5 \times 37 \mathrm{mJy}^{-1}$ beam $^{-1}$ (the rms noise). The position of 49 Ceti is marked with a star symbol, while the green line indicates the position angle derived by Wahhaj et al. (2007) from mid-IR imaging. The contour colors indicate heliocentric line-of-sight velocity; the four distinct velocities shown are 9.0, $11.1,13.2$, and $15.3 \mathrm{~km} \mathrm{~s}^{-1}$, in the order of bluest to reddest channel. No emission was detected outside this velocity range.

and the passband calibrators were the quasars 3C 454.3 and $\mathrm{J} 0530+135$. Gain calibration was carried out using the quasar J0132-169, located just $1.3^{\circ}$ from 49 Ceti; the flux derived for this quasar was $0.93 \mathrm{Jy}$. The nearby quasar J0006-063 was also included to test the quality of the phase transfer from J0132-169.

Two $2 \mathrm{GHz}$ sidebands separated by $10 \mathrm{GHz}$ were used, yielding a continuum sensitivity of $0.7 \mathrm{mJy}(1 \sigma)$. Spectral resolution in the line was $0.26 \mathrm{~km} \mathrm{~s}^{-1}$, subsequently binned to $2.1 \mathrm{~km} \mathrm{~s}^{-1}$, with rms sensitivity $0.030 \mathrm{Jy}$ in a single $2.1 \mathrm{~km} \mathrm{~s}^{-1}$ channel. The local standard of rest (LSR) velocities were converted to heliocentric using an offset of $-9.14 \mathrm{~km} \mathrm{~s}^{-1}$. The synthesized naturally weighted beam in the $\operatorname{CO} J=2-1$ line was $1.0^{\prime \prime} \times 1.2^{\prime \prime}$, at a position angle of $-78.6^{\circ}$. Imaging was carried out using the MIRIAD software package.

\section{RESULTS AND ANALYSIS}

Figure 1 shows the observed line emission from the region around 49 Ceti. Four velocity channels are shown, with the velocity indicated by the color of the contour lines. The observations are centered on the $\mathrm{J} 2000.0$ coordinates of 49 Ceti; the star symbol indicates the position corrected for the proper motion measured by Hipparcos. The maximum $\mathrm{S} / \mathrm{N}$ in the line is 8 . The $\mathrm{CO} J=2-1$ emission appears to be in an extended rotating structure of $>2$ " radius, apparently viewed close to edge-on. The symmetric distribution of the emission in the four velocity channels implies a heliocentric velocity near $12.2 \mathrm{~km} \mathrm{~s}^{-1}$, consistent with previous determinations of the systemic velocity $\left(10.5\right.$ and $9.9 \mathrm{~km} \mathrm{~s}^{-1}$ for the disk and the star, respectively; see Dent et al. [2005] and references therein). No emission is detected outside the range of velocities shown. The wide separation of the emission peaks, combined with a lack of compact, high-velocity emission, suggests that the central regions are clear of $\mathrm{CO} J=2-1$ emission out to $\sim 90$ AU radius $\left(\sim 1.5^{\prime \prime}\right)$, twice that of the larger ring predicted 
TABLE 1

Observational Parameters for 49 Ceti

\begin{tabular}{|c|c|c|c|c|}
\hline Parameter & ${ }^{12} \mathrm{CO}(3-2)^{\mathrm{a}}$ & ${ }^{12} \mathrm{CO}(2-1)$ & ${ }^{13} \mathrm{CO}(2-1)$ & Continuum \\
\hline 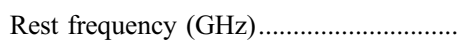 & 345.796 & 230.538 & 220.399 & $230.5(\mathrm{USB})^{\mathrm{b}}$ \\
\hline 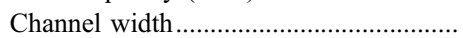 & $0.27 \mathrm{~km} \mathrm{~s}^{-1}$ & $2.1 \mathrm{~km} \mathrm{~s}^{-1}$ & $8.4 \mathrm{~km} \mathrm{~s}^{-1}$ & $2 \times 2 \mathrm{GHz}$ \\
\hline Beam size $(\mathrm{FWHM})(\operatorname{arcsec}) . . . \ldots \ldots \ldots \ldots \ldots . . . . .$. & $14^{\prime \prime}$ & $1.0^{\prime \prime} \times 1.2^{\prime \prime}$ & $1.0^{\prime \prime} \times 1.2^{\prime \prime}$ & $1.0^{\prime \prime} \times 1.2^{\prime \prime}$ \\
\hline P.A. $(\operatorname{deg})$ & $\ldots$ & -78.6 & -78.6 & -78.6 \\
\hline 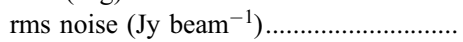 & 0.22 & 0.030 & 0.017 & $7.0 \times 10^{-4}$ \\
\hline 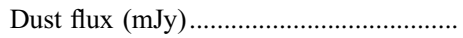 & $\ldots$ & $\ldots$ & $\ldots$ & $<2.1$ \\
\hline Peak brightness temperature $(\mathrm{K}) \ldots \ldots \ldots \ldots$ & $0.076 \pm 0.008$ & $3.5 \pm 0.5$ & $<0.8$ & $\ldots$ \\
\hline Integrated intensity $\left(\mathrm{Jy} \mathrm{km} \mathrm{s}^{-1}\right) \ldots \ldots \ldots \ldots \ldots$ & $9.5 \pm 1.9$ & $2.0 \pm 0.3$ & $<0.5$ & $\ldots$ \\
\hline
\end{tabular}

a Dent et al. (2005)

b Upper sideband frequency; lower sideband is centered at $220.5 \mathrm{GHz}$. Both sidebands have $2 \mathrm{GHz}$ width.

from the $\mathrm{CO} J=3-2$ single-dish data. Table 1 lists the observed parameters of the system.

Assuming optically thin lines and local thermodynamic equilibrium (LTE), the total mass in CO probed by the $J=2-1$ transition is given by

$$
M=\frac{4 \pi}{h \nu_{21}} \frac{F_{21} m d^{2}}{A_{21} x_{2}}
$$

where the subscript " 21 " refers to the $\mathrm{CO}(2-1)$ transition, $F$ is the integrated flux in the line, $d$ is the distance to the source (61 pc; Hipparcos), $m$ is the mass of the CO molecule, $\nu$ is the rest frequency of the transition, $h$ is Planck's constant, and $x_{2} \equiv N_{2} / N_{\text {tot }}$ where $N_{2}$ is the population in the $J=2$ rotational level while $N_{\text {tot }}$ is the total $\mathrm{CO}$ population. The $\mathrm{CO}$ mass calculated using this method is $2.2 \times 10^{-4} M_{\oplus}$. Using the canonical $\mathrm{CO} / \mathrm{H}_{2}$ ratio of $10^{-4}$, this yields a molecular hydrogen mass of $2.2 M_{\oplus}$, consistent with the value of $6.3 \times 10^{-3} M_{\text {Jup }}=2.0 M_{\oplus}$ calculated by Zuckerman et al. (1995).

No continuum emission was detected at this combination of resolution and sensitivity. This indicates one of two things: either the continuum flux is concentrated at the center of the disk but the total flux is too low to be detected, or the total flux may be larger but spread over many beams, so that the brightness within each beam is below our detection threshold. These observations were sensitive enough to detect the higher continuum flux reported by Bockelée-Morvan et al. (1994) if it were concentrated within a few synthesized beams. However, an extrapolation of the Song et al. (2004) value for a typical circumstellar dust spectrum predicts a lower flux by a factor of 6 , which is just below the detection threshold. The lack of an SMA continuum detection at $230 \mathrm{GHz}$ is therefore inconclusive: if the Song et al. (2004) value is correct, we would not expect to detect even centrally concentrated emission, so we cannot constrain the spatial extent of dust emission through the nondetection at $230 \mathrm{GHz}$.

\section{DISK MODELING}

In order to gain insight into the physical processes at work in the 49 Ceti system, we carried out modeling of the disk with COSTAR (Kamp \& Bertoldi 2000; Kamp \& van Zadelhoff 2001), a code that solves the chemical equilibrium simultaneously with a detailed heating and cooling balance to determine gas properties of circumstellar disks. In the following, the salient features of these models are summarized. The chemistry is modeled using a network of 48 different species covering the elements $\mathrm{H}$, $\mathrm{He}, \mathrm{C}, \mathrm{O}, \mathrm{S}, \mathrm{Mg}$, Si, and Fe. The elemental abundances and key parameters of these models, including the stellar mass, radius, effective temperature, surface gravity, and ultraviolet flux, are summarized in Table 2. The 48 species are connected through 281 reactions, including cosmic-ray chemistry, photochemistry, and the chemistry of excited $\mathrm{H}_{2}$. We compute equilibrium chemistry using a modified Newton-Raphson algorithm. The solution then only depends on the element abundances and not on initial conditions.

We use the results of dust modeling by Wahhaj et al. (2007) and assume large $30 \mu \mathrm{m}$ blackbody grains with radiative efficiencies of $Q_{\lambda}=2 \pi a / \lambda$ for $\lambda>2 \pi a$ and $Q_{\lambda}=1$ otherwise. These grains are efficient absorbers and inefficient emitters, thus achieving dust radiative equilibrium temperatures of

$$
T_{\text {dust }}=324\left(\frac{L_{*}}{L_{\odot}}\right)^{0.2}\left(a_{\mu \mathrm{m}}\right)^{-0.2}\left(r_{\mathrm{AU}}\right)^{-0.4} \mathrm{~K} .
$$

Here $L_{*}$ and $L_{\odot}$ are the stellar and solar luminosity respectively, $a_{\mu \mathrm{m}}$ is the grain size in microns, and $r_{\mathrm{AU}}$ is the distance from the star in astronomical units. The gas temperature is derived from a detailed energy balance including the most relevant heating and cooling processes (Kamp \& van Zadelhoff 2001).

The radiation field consists of both stellar and interstellar components. The stellar properties are determined by a Kurucz model fit to photometric points collected from the literature (Wahhaj et al. 2007; Sylvester et al. 1996; Bockelée-Morvan et al. 1994; Song et al. 2004); using $T_{\text {eff }}=10,000 \mathrm{~K}$ and $\log g=4.5$, consistent with the values quoted by Chen et al. (2006), the derived stellar luminosity is $L_{*}=26.1 L_{\odot}$ and the radius is $1.7 R_{\odot}$. The SED and Kurucz model are plotted in Figure 2, including dereddening according to extinction derived by Sylvester et al.

TABLE 2

Element Abundances and Parameters

\begin{tabular}{|c|c|}
\hline Parameter $^{\mathrm{a}}$ & Value \\
\hline$A_{\mathrm{He}} \cdots \cdots \cdots \cdots \cdots$ & $7.5 \times 10^{-2}$ \\
\hline 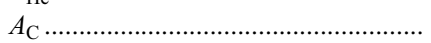 & $1.3 \times 10^{-4}$ \\
\hline$A_{\mathrm{O}}$ & $2.9 \times 10^{-4}$ \\
\hline 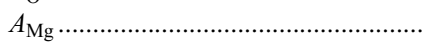 & $4.2 \times 10^{-6}$ \\
\hline 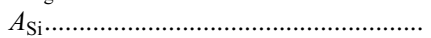 & $8.0 \times 10^{-6}$ \\
\hline$A_{\mathrm{S}}$ & $1.9 \times 10^{-6}$ \\
\hline 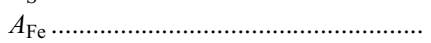 & $4.3 \times 10^{-6}$ \\
\hline 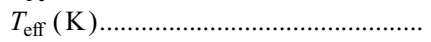 & 10,000 \\
\hline $\log g$ & 4.5 \\
\hline 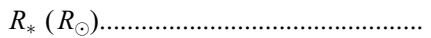 & 1.7 \\
\hline 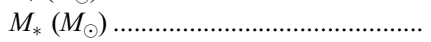 & 2.3 \\
\hline$\sigma_{\mathrm{UV}}\left(\mathrm{cm}^{-2} \mathrm{H}_{\text {-atom }}{ }^{-1}\right) \ldots \ldots \ldots \ldots \ldots \ldots \ldots \ldots \ldots \ldots \ldots$ & $4.68 \times 10^{-24}$ \\
\hline
\end{tabular}
USEd In The Disk Models

a Gas-phase abundances $(A)$ are relative to hydrogen. 


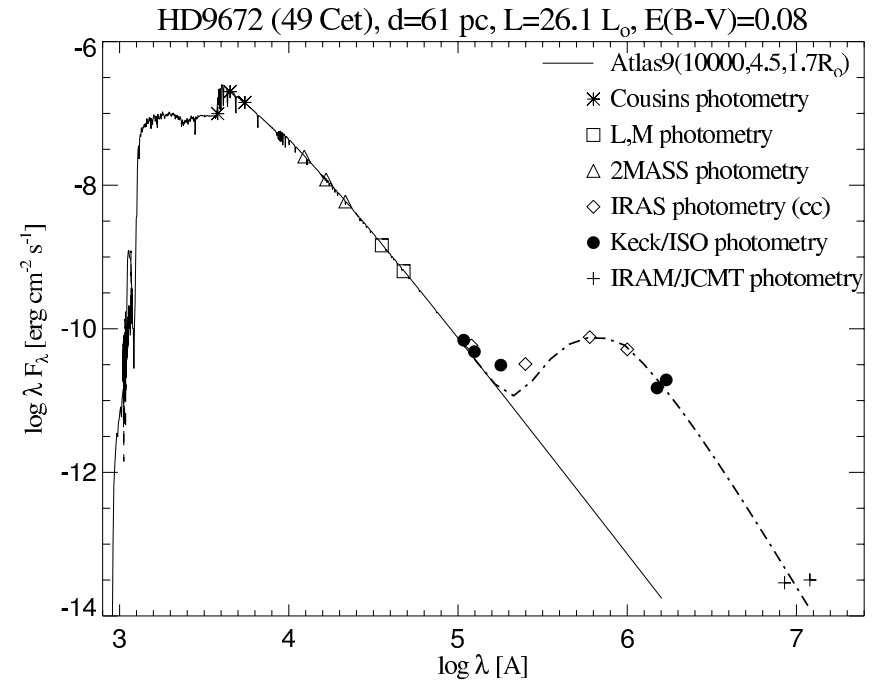

FIG. 2.-SED (dereddened according to extinction derived by Sylvester et al. [1996] and Cardelli et al. [1989] extinction law) for 49 Ceti using available optical, infrared, and submillimeter photometry. The solid line denotes a Kurucz stellar atmosphere model fitted to the photometry using the Hipparcos distance of $61 \mathrm{pc}$. The dot-dashed line shows the SED for the best-fit model of the outer disk (see $\S 4.4$ for details).

(1996) and using a Cardelli et al. (1989) extinction law. The solid line in the figure denotes the fit to the photometry of a Kurucz stellar atmosphere model at the Hipparcos distance of $61 \mathrm{pc}$. The dashed line shows the SED of the best-fit model of the outer disk as described in $\S 4.5$. The interstellar radiation field in the ultraviolet is assumed to be $1.2 \times 10^{7} \mathrm{~cm}^{-2} \mathrm{~s}^{-1}$ (Habing 1968).

A basic model of the dust disk was constructed according to the Bayesian analysis of mid-infrared emission carried out by Wahhaj et al. (2007). Their model consists of an inner disk extending from 30 to $60 \mathrm{AU}$, composed primarily of small grains $(a \sim 0.1 \mu \mathrm{m})$ with a surface density of $5 \times 10^{-8} \mathrm{~g} \mathrm{~cm}^{-2}$, and an outer disk extending from 60 to 900 AU composed of larger grains $(a \sim 15 \mu \mathrm{m})$ with a surface density of $3 \times 10^{-6} \mathrm{~g} \mathrm{~cm}^{-2}$. They derive a surface density distribution for the outer disk that is constant with radius, yielding a total disk mass of $0.35 M_{\oplus}$. From the mid-IR images, they also determine a position angle of $125^{\circ} \pm 10^{\circ}$ (indicated in Fig. 1) and an inclination of $60^{\circ} \pm 15^{\circ}$. We use this model as a starting point for the disk structure, since it reflects the best available information on the dust density distribution. However, since the molecular gas emission provides better constraints on some aspects of disk structure, including the vertical density distribution and the surface density structure of the outer disk, we introduce refinements to this initial model where justified, as described in $\S \S 4.2$ and 4.4 below. For the large grain population, our model uses $30 \mu \mathrm{m}$ grains instead of $15 \mu \mathrm{m}$ grains, although the grain size used in this simple model is highly degenerate with other disk properties, as discussed in $\S 4.4$.

To predict gas properties from this dust model, we make two primary assumptions: (1) gas and dust are well mixed, (2) the gas : dust mass ratio is constant. We initially assume a constant scale height $H=2 \mathrm{AU}$, since there is no information on disk scale height from the dust model of Wahhaj et al. (2007); we also begin by retaining the inner and outer radii and radially constant surface density structure from the Wahhaj et al. (2007) model, although these assumptions are modified in $\S$ 4.2. Throughout the modeling process, we use the canonical gas : dust mass ratio of 100 and assume that the disk is embedded in interstellar ma- terial of density $10 \mathrm{~cm}^{-3}$ to avoid model densities dropping to unrealistically low values near the boundaries of the numerical grid.

To compare our models with the SMA data, we use the radiative transfer code RATRAN (Hogerheijde \& van der Tak 2000) to generate a sky-projected image of the $\mathrm{CO} J=2-1$ emission predicted for the physical model. We then use the MIRIAD task uvmodel to sample the image with the combination of spatial frequencies and visibility weights appropriate for our SMA data. We allow the inclination and position angle of the system to vary in order to best match the data.

\subsection{Inner Disk}

In the inner disk, inside $60 \mathrm{AU}$, composed primarily of small grains, the stellar radiation field raises the dust temperature to $1000-2000 \mathrm{~K}$ and dissociates most of the molecular gas. In this region, the dominant form of carbon is $\mathrm{C}^{+}$, and even hydrogen is predominantly atomic. We therefore ignore the inner disk component in subsequent modeling and focus on reproducing the observed CO emission with only the outer disk component.

This lack of molecular gas in the inner disk is consistent with the nondetection of warm $\mathrm{H}_{2}$ by Chen et al. (2006) and Carmona et al. (2008) and with the lack of high-velocity CO emission in Figure 1. The lack of $\mathrm{CO}$ emission more than $4.3 \mathrm{~km} \mathrm{~s}^{-1}$ from the stellar velocity is consistent with an absence of $\mathrm{CO}$ within a radius of $\sim 90 \mathrm{AU}$, for gas in Keplerian rotation around a star of $2.3 \mathrm{M}_{\odot}$.

\subsection{Outer Disk}

There are three primary features of the observed CO emission from the outer disk that we attempted to reproduce with this modeling effort: (1) the separation of the emission peaks in the outer channels $\left(\sim 3^{\prime \prime}\right),(2)$ the spatial extent of the $\mathrm{CO}$ emission in all channels, and (3) the strength of the emission. Reproducing these features of the observed $\mathrm{CO}$ emission requires several modifications to the best-fit Wahhaj et al. (2007) model of the outer dust disk, including adjustments to the inner and outer radii and a departure from the constant surface density prescription.

At first glance, the inner radius of 60 AU derived by Wahhaj et al. (2007) might seem consistent with the lack of emission within $90 \mathrm{AU}$ derived from the missing high-velocity wings in our data; however, there is a large region at the inner edge of the outer disk subject to photodissociation by stellar radiation, which therefore contributes little to the $\mathrm{CO}$ emission. In order to reproduce the separation of the emission peaks, material is required inside this $60 \mathrm{AU}$ radius. We therefore take the uncertainties in the Wahhaj et al. (2007) dust distribution into account and allow the inner disk radius to vary. However, moving the inner radius closer than $\sim 40$ AU to the star results in high-velocity emission that we do not observe in the data, while still producing emission peaks wider than observed. We therefore set the disk inner radius at $40 \mathrm{AU}$ and then adjust the gas densities to further reduce the separation of the emission peaks.

Increasing the total gas mass leads to an elongated morphology with an aspect ratio larger than the observations, as the optical depth rises throughout the disk. To meet the three criteria of (1) enough gas-phase $\mathrm{CO}$ near the inner disk edge to reproduce the observed peak separation, (2) low enough optical depth in the outer parts of the disk to keep the emission from becoming more elongated than the data (through photodissociation by interstellar UV photons), and (3) maintaining an inner radius large enough to avoid generating high-velocity emission that is not present in the data, we must "pile up" material at the inner disk edge to enhance shielding and concentrate emission. We therefore modify the initial assumption of constant surface density as derived from 
the infrared analysis, instead adopting an $r^{-\epsilon}$ density profile. We simultaneously relax the constant scale height assumption, introducing a scale height $H$ that increases linearly with radius $r$, with proportionality constant $h=H / r$. The full two-dimensional density structure then becomes $n(r, z)=r^{-\epsilon} \exp \left(-z^{2} / 2 H^{2}\right)$, where the exponent $\epsilon$ and scale height constant $h$ are varied to obtain the best fit to the $\mathrm{CO}$ data.

The power-law surface density profile results in a much better match between the model and the observed emission-peak separation. It also curbs the elongation of the emission to some extent, as the vertical column density of the outer disk drops and the material far from the star becomes subject to dissociation by interstellar radiation. However, even steep power-law indices for the surface density profile do not result in a completely photoevaporated outer disk and consequently produce emission that is much more elongated than observed. In a next step, we therefore reduce the outer radius from 900 to $200 \mathrm{AU}$. While this is at the lower end of the range allowed by Wahhaj et al. (2007), their derived outer radius was based largely on the uncertain millimeter flux measurement, and the gas geometry is likely a better probe of the disk extent.

\subsection{Grid of Disk Models}

After these initial studies of the outer disk, it became clear that several model parameters were ill constrained by previously existing data. Specifically, the disk mass is constrained only by the weakly detected and contradictory millimeter flux measurements; similarly, the density power-law index $\epsilon$ is ill determined by the infrared observations, which are primarily sensitive to inner disk emission. The scale height $h$ is also completely unconstrained by the continuum or single-dish measurements, neither of which is sensitive to disk structure in the vertical direction. The disk geometry (P.A. and inclination) quoted by Wahhaj et al. (2007) is also subject to large uncertainties, due to the irregular shape of the emission observed in the infrared. We therefore attempt to better constrain these disk parameters by using our resolved CO gas-line observations. Gas lines are generally more sensitive than dust emission to temperature and density gradients and can thus provide means to break model degeneracies. We ran grids of models for the three structural parameters (disk mass, density index, scale height) and two geometrical parameters (P.A., inclination), finding the best-fit values by calculating and minimizing a $\chi^{2}$ value comparing the model to the observed emission from the disk. Due to the computational intensity of the calculations necessary to determine the chemistry and radiative transfer solutions for each model, we ran only a sparsely sampled grid of models. In order to ensure that the final model reflects all available observational constraints, we centered the grid on the fiducial model of $\S \S 4$ and 4.2 adjusted the parameters only as necessary to better reproduce the new $\mathrm{CO}(2-1)$ observations, moving from coarse to fine grids to ensure adequate exploration of the parameter space. We use the modeling primarily as a demonstration that the basic features of the observed $\mathrm{CO}$ emission can be reproduced by a simple azimuthally symmetric model of disk structure; the "best-fit" model should therefore be viewed as representative of an initial understanding of the features of the system rather than as a conclusive determination of the disk structural parameters.

\subsubsection{CO Chemistry Across the Model Grid}

The $\mathrm{CO}$ chemistry is dominated by photodissociation in a number of UV bands and thus the abundance of $\mathrm{CO}$ in each model is mostly dependent on the radial and vertical column densities being able to shield the stellar and interstellar UV ra- diation, respectively. In the following we briefly discuss some basic characteristics of the model grid.

The surface density in the models is independent of the scale height and hence the radial mass distribution in each model can be written as $M(R) \propto R^{-\epsilon+3}$, where $M(R)$ denotes the mass inside a radius $R$. So, as we increase the density power-law exponent $\epsilon$, the inner region of the outer disk harbors a larger fraction of the total mass. The densities in this region of the disk become higher and hence it is easier to obtain the critical column densities necessary for UV shielding in the radial direction. However, a shallower gradient for the density distribution translates into higher densities in the outer parts of the disk, thus enhancing the vertical shielding in the outer disk compared to models with high $\epsilon$. None of our models is optically thick in the dust continuum, so the UV shielding is mainly $\mathrm{H}_{2}$ shielding of the $\mathrm{CO}$ bands due to their overlap in wavelengths; $\mathrm{CO}$ self-shielding also plays a role.

With this basic picture, we can understand the CO chemistry displayed in Figure 3 as a function of disk mass (right column) and density gradient $\epsilon$ (middle column). As the total disk mass is increased, CO first starts to build up in the radial direction. It can still be dissociated by the vertically impinging interstellar UV radiation field in the outer regions of the disk (150-200 AU) until the disk reaches a mass of $\sim 17 M_{\oplus}$, at which point it becomes opaque in the $\mathrm{CO}$ bands even in the vertical direction. A shallow density gradient always leads to smaller radial column densities at the same reference radius, thus pushing the $\mathrm{C}^{+} / \mathrm{C} / \mathrm{CO}$ transition farther out in radial distance. In our best-fit model of $13 M_{\oplus}$, a change in $\epsilon$ from 2.5 to 1.1 changes the radius for the $\mathrm{C}^{+} / \mathrm{C} / \mathrm{CO}$ front from close to $40 \mathrm{AU}$ to $190 \mathrm{AU}$.

The scale height $h$ of the models affects only the vertical density structure in the models. However, since density and chemistry are closely intertwined, it can strongly impact the overall radial and vertical structure of the CO chemistry. From a comparison of the center panel with the bottom left panel in Figure 3, we see that a factor of 2 lower scale height with respect to the best-fit model ( $h=0.02)$ enhances the CO abundance in the disk significantly, leading to radial and vertical column densities that are more than a factor of 10 higher with respect to the best-fit model. The total CO mass increases by a factor of 10 as well, with the integrated emission undergoing a corresponding dramatic increase.

Table 3 displays some key results from a subset of grid models, such as characteristic radial and vertical CO column densities, CO masses, and total CO $J=2-1$ line emission. For all models in the table, the inner radius is fixed at $40 \mathrm{AU}$ and the outer radius at $200 \mathrm{AU}$.

\subsubsection{From Chemistry to Observables}

The predicted CO $J=2-1$ emission for the models in Figure 3 is displayed in Figure 4; a comparison of these figures illustrates the ways in which differences in chemical structure are manifested in the observable properties of the $\mathrm{CO}$ emission. The $\mathrm{CO}$ emission is sampled with the same spatial frequencies and visibility weights as the SMA data and displayed in renzogram form with the same velocity structure as in Figure 1. In order to emphasize the relative structural differences between models, the contour levels are $15 \%$ of the peak flux for each model, with the absolute flux indicated by the thickness of the contours and also printed explicitly at the top of each panel.

The decreased shielding in the inner disk caused by reducing the density gradient $\epsilon$ is visible as a lengthening of the emission in the central channels and a widening of the emission peaks in the outer channels in the low- $\epsilon$ model (bottom center panel). 

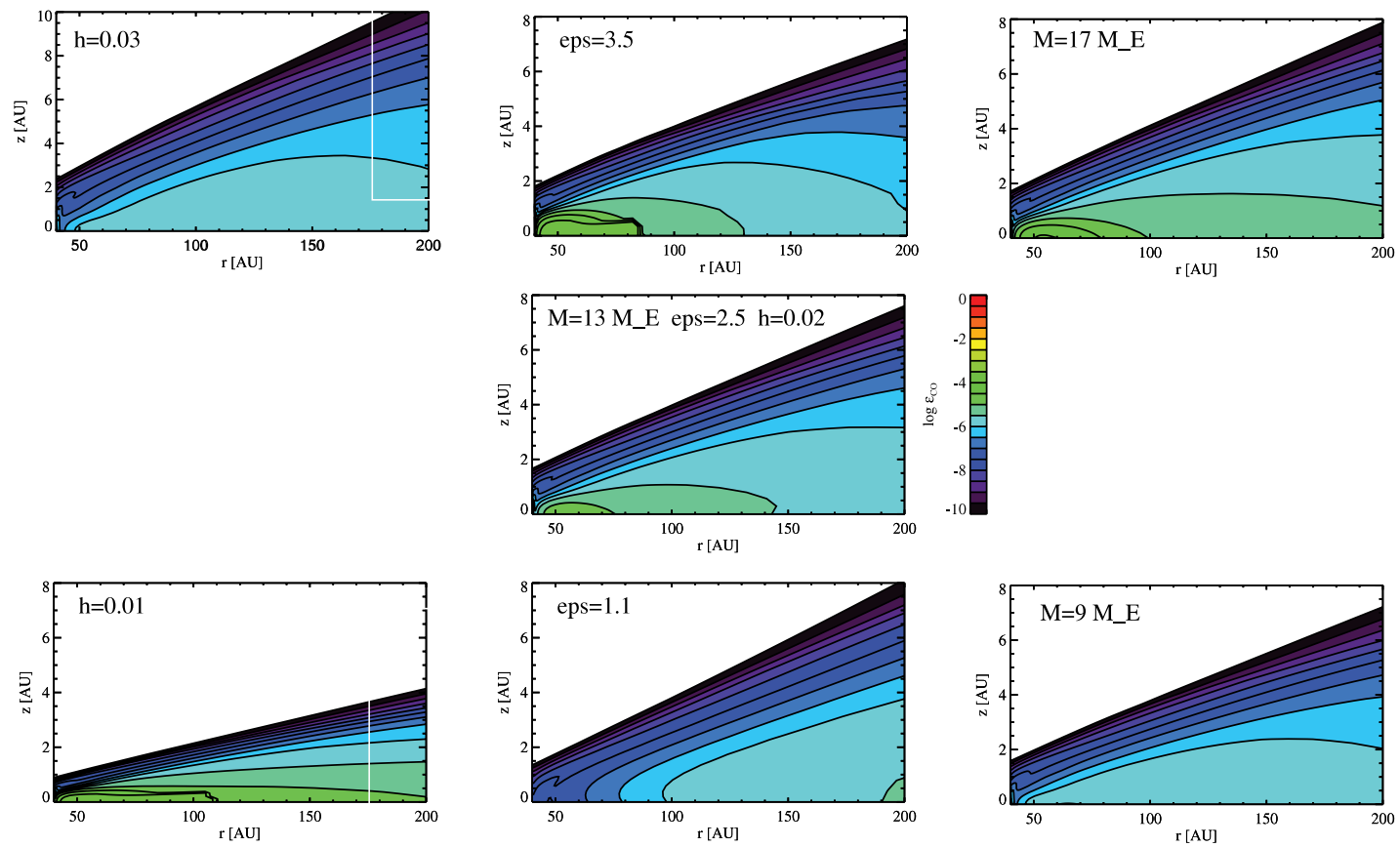

FIG. 3.- Two-dimensional CO abundances in a subset of disk models. The center panel shows the best-fit model $\left(M=13 M_{\oplus}, \epsilon=2.5, h=0.02\right)$, while the rows of models above and below show the effects of incrementing and decrementing, respectively, each of the three structural parameters that were allowed to vary during the fitting process: $h$ (left column), $\epsilon$ (center column), and $M_{\text {disk }}$ (right column). The values of the parameters shown are $h=0.01,0.03 ; \epsilon=1.1,3.5 ;$ and $M=9,17 M_{\oplus}$.

Increasing $\epsilon$ (top center panel) leads to enhanced shielding at the disk inner edge, causing much higher $\mathrm{CO}$ fluxes in the outer part of the disk and extremely high contrast between the inner and outer velocity channels.

The primary observable consequence of adjusting the mass (right panels) is that the increased or decreased shielding from extra gas leads to a corresponding increase or decrease in the total CO flux; changes to the shape of the emission are minimal, and the primary difference between models of different mass over the mass range under consideration is simply in the relative brightness of the emission.

Differences in the scale height of the disk similarly manifest as differences in the flux scale; however, decreasing the scale height (bottom left panel) also causes greater shielding at the inner disk edge, leading to greater elongation of the outer velocity channels and causing the inner velocity channels to draw together and overlap as the CO flux rises throughout the inner areas of the disk. An increase in scale height (top left panel) leads to a greater area in the front and back of the disk, projected along our line of sight, which increases the flux in the central channels and leads to a lower contrast between the inner and outer channels of the disk.

\subsection{Spectral Energy Distribution}

After converging initially on a model that was able to reproduce the observed $\mathrm{CO} J=2-1$ emission, we used that model to

TABLE 3

Derived Quantities from a Subset of the 49 Ceti Disk Models

\begin{tabular}{|c|c|c|c|c|c|c|}
\hline $\begin{array}{c}M_{\text {disk }}{ }^{\mathrm{a}} \\
\left(M_{\oplus}\right)\end{array}$ & $\epsilon$ & $h$ & $\begin{array}{l}N(\mathrm{CO})_{\text {radial }}{ }^{\mathrm{b}} \\
\left(10^{18} \mathrm{~cm}^{-2}\right)\end{array}$ & $\begin{array}{c}N(\mathrm{CO})_{\mathrm{vertical}}^{100 \mathrm{AU}} \mathrm{c} \\
\left(10^{15} \mathrm{~cm}^{-2}\right)\end{array}$ & $\begin{array}{c}M_{\mathrm{CO}}{ }^{\mathrm{d}} \\
\left(10^{-4} M_{\oplus}\right)\end{array}$ & $\begin{array}{l}I_{\mathrm{CO}}(J=2-1)^{\mathrm{e}} \\
\left(\mathrm{Jy} \mathrm{km} \mathrm{s}^{-1}\right)\end{array}$ \\
\hline $13 \ldots \ldots \ldots \ldots$ & 2.5 & 0.020 & 2.76 & 4.23 & 9.66 & 2.6 \\
\hline 9 & 2.5 & 0.020 & 0.32 & 1.82 & 2.46 & 1.2 \\
\hline 17 & 2.5 & 0.020 & 13.5 & 9.06 & 37.2 & 6.9 \\
\hline $13 \ldots \ldots$ & 3.5 & 0.020 & 15.1 & 4.47 & 98.0 & 11.7 \\
\hline $13 \ldots \ldots \ldots \ldots \ldots \ldots \ldots$ & 1.1 & 0.020 & 0.13 & 0.91 & 3.74 & 2.3 \\
\hline 13 & 2.5 & 0.010 & 42.8 & 78.4 & 96.6 & 14.5 \\
\hline 13 & 2.5 & 0.030 & 0.12 & 2.20 & 2.97 & 1.5 \\
\hline
\end{tabular}

a Total disk gas mass.

b Total radial CO column density through the midplane.

c CO vertical column density at $100 \mathrm{AU}$.

$\mathrm{d}$ Total CO mass in the disk.

e Integrated $\operatorname{CO}(J=2-1)$ line emission. 

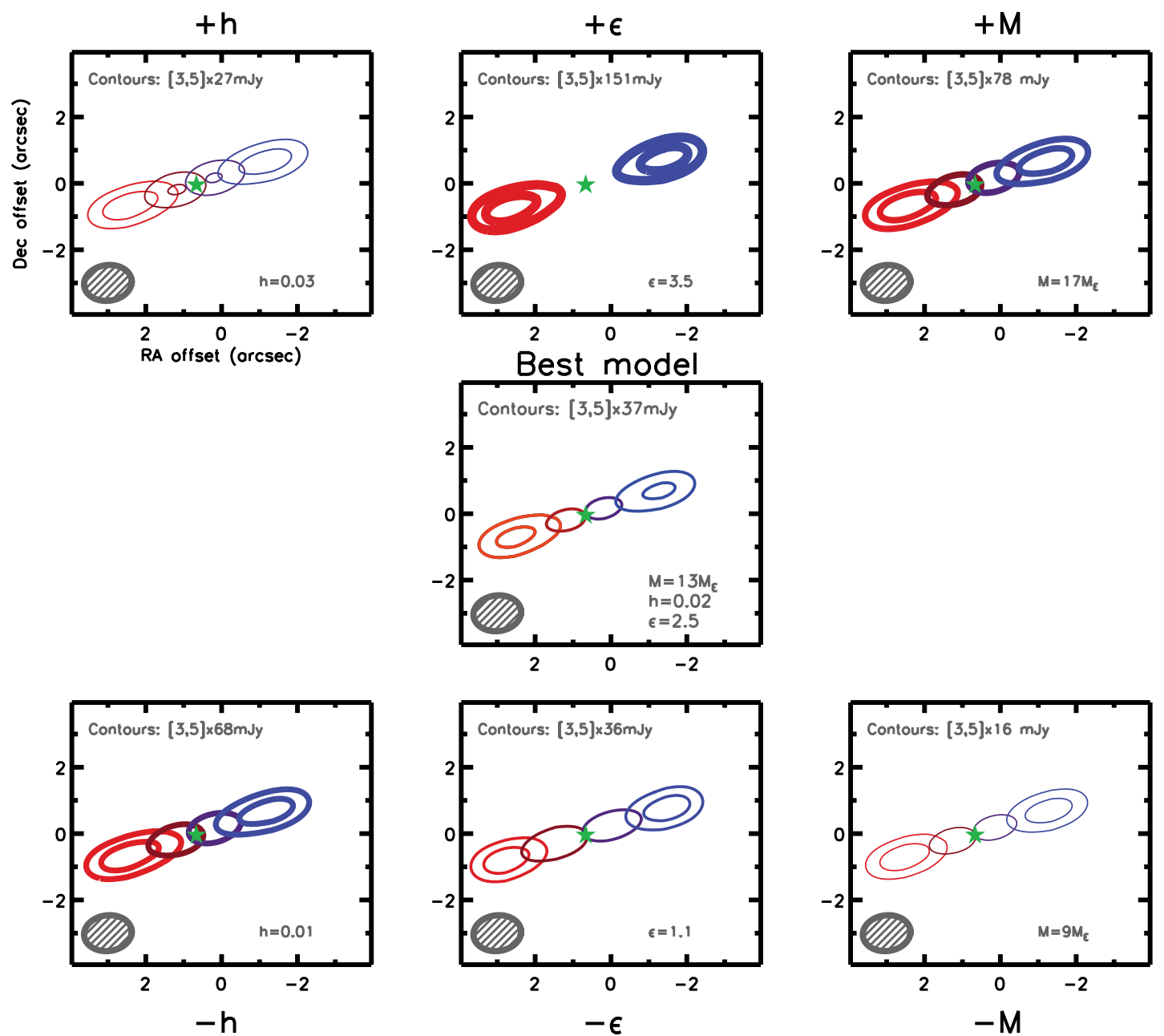

FIG. 4. $-\mathrm{CO} J=2-1$ emission predicted for the subset of models shown in Fig. 3, sampled with the same spatial frequencies and visibility weights as the SMA data in Fig. 1. The center panel shows the best-fit model, while the rows of models above and below show the effects of incrementing and decrementing, respectively, each of the three structural parameters that we allowed to vary during the fitting process: $h$ (left column), $\epsilon$ (center column), and $M_{\text {disk }}$ (right column). The contour levels are displayed in the upper left corner of each panel; they are set at $3 \times 15 \%$ and $5 \times 15 \%$ of the peak flux for each model. The thickness of the contours is proportional to the absolute flux: thicker contours indicate that the source is brighter than the data, while thinner contours indicate that it is fainter than the data. The contour levels in the center panel are identical to those in Fig. 1. Table 4 gives the full list of parameters for the best-fit model.

predict the SED. This serves as an a posteriori test of the consistency between the gas and dust properties in the models and the available observables.

We integrate over the disk volume to obtain the flux as a function of wavelength,

$F_{\lambda}=\left(\frac{\pi a^{2}}{d^{2}}\right) \iint 2 \pi r B_{\lambda}\left[T_{\text {dust }}(r, z)\right] n_{\text {dust }}(r, z) Q(\lambda) d z d r$

where $d$ is the distance to the source and $n_{\text {dust }}$ is the number density of dust grains in $\mathrm{cm}^{-3}$. We assume throughout a grain density of $2.5 \mathrm{~g} \mathrm{~cm}^{-3}$.

While the predicted shape of the SED matches the observations well, the absolute fluxes are too high by a factor of $\sim 5$. Adjusting the temperature of the dust grains alters the shape of the SED curve, causing it to deviate from the observed shape; we were therefore required to increase the gas : dust ratio from 100 to 500 in order to reproduce the observed photometry. This unusually high ratio is likely an artifact of the simple assumptions of the model, since little information is available about the dust distribution in this system (and none at all from our data). For example, the mass of the system is likely not all in $30 \mu \mathrm{m}$ grains, and a significant fraction of the mass may be in larger grains that contribute little to the infrared emission. Another possibility is that the overall gas : dust ratio is consistent with the standard value, but that gas and dust are not well mixed: for example, much of the excess emission may arise from the inner edge of the disk, which will be directly illuminated and heated by the stellar radiation. Resolved observations of the dust continuum emission would test this hypothesis by placing constraints on the spatial distribution of the emitting region. Including effects such as this would significantly complicate the model presented here, as the $\mathrm{H}_{2}$ formation rate would be affected by varying the abundance of the dust on which it forms. In general, the dust size and gas : dust mass ratio are strongly related by the total dust surface required to maintain the observed quantity of molecular gas; these are in turn dependent on the stellar properties determining dust grain temperatures. None of these dust-dependent quantities are well constrained by available data. Given the observations available and the extremely simplified dust model, which not only neglects the size distribution but also the possibility of a mixture of compositions and opacities, we use the simplest assumption of an altered gas : dust ratio in order to 
TABLE 4

Parameters for Best-Fit Disk Model

\begin{tabular}{|c|c|}
\hline Parameter & Value \\
\hline$h \ldots$ & $0.020_{-0.005}^{+0.015}$ \\
\hline$\epsilon \ldots \ldots$ & $2.5_{-1.0}^{+0.005}$ \\
\hline 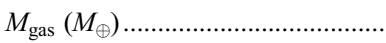 & $13 \pm 3$ \\
\hline$M_{\text {dust }}\left(M_{\oplus}\right) \ldots \ldots \ldots \ldots \ldots \ldots$ & $0.02 \pm 0.01$ \\
\hline$i(\operatorname{deg}) \ldots \ldots \ldots \ldots$ & $90 \pm 5$ \\
\hline P.A. (deg) .. & $-70 \pm 10$ \\
\hline$R_{\text {in }}(\mathrm{AU})^{\mathrm{a}} .$. & 40 \\
\hline$R_{\text {out }}(\mathrm{AU})^{\mathrm{a}}$ & 200 \\
\hline
\end{tabular}

${ }^{\text {a }}$ For a description of the constraints on the inner and outer radii, see $\S 4.2$.

conduct a consistency check of the temperature and density structure of the gas model.

Decreasing the total dust mass in the model to match the SED reduces the grain surface area for $\mathrm{H}_{2}$ formation. Thus, molecular hydrogen begins to form at larger radii and greater depth, compared to the initial model with the canonical gas : dust ratio of 100. As a consequence of less effective UV shielding, the total $\mathrm{CO}$ mass decreases. Hence, the total mass of the best-fit model has to be increased slightly to compensate for the lower molecular gas fraction. As a secondary effect, the overall gas temperature of the dust-depleted model also decreases due to the diminished photoelectric heating in the disk. The corresponding SED predicted for these parameters is indicated by the dot-dashed line in Figure 2. The mid-infrared flux points are underestimated by this model because we do not include the inner disk component of Wahhaj et al. (2007); as our data provide no constraints on the properties of the inner disk, we ignore this component and concentrate on the fit to the outer disk. The flux predicted by the model SED is consistent with our own continuum upper limit reported in Table 1.

\subsection{Best-Fit Disk Model}

The Figure 4 (center panel) shows the best-fit model from the grid, with the minor modifications introduced by reproducing simultaneously the SED. The structural and geometric parameters for this model are listed in Table 4. The errors given in the table are the approximate $1 \sigma$ uncertainty range interpolated from the $\chi^{2}$ grid.

This model reproduces the basic features of the $\mathrm{CO} J=2-1$ emission well, including the strength of the emission, the separation of the emission peaks, and the spatial extent of the emission. There are still several important differences between the model and the data, however, including (1) an inability to reproduce the changes in position angle with radius evident in the data (the "wings" of emission extending to the southeast and northwest of the position angle axis), and (2) the separation of the innermost, low-velocity channels. Both of these may be indicative of departures from azimuthal symmetry in the disk structure, the former possibly indicating a warp in the disk and the latter apparently pointing to a deficit of emission along the minor axis of the disk. In none of our models were we able to reproduce the wide separation between the inner channels; while the $\mathrm{S} / \mathrm{N}$ in these channels is low, the observed CO morphology is difficult to reproduce in detail with a simple, azimuthally symmetric disk model. The $\mathrm{CO}$ emission for this best-fit model is optically thin in both the $J=2-1$ and $J=3-2$ transitions, even for the edgeon disk orientation, and therefore traces the full column density of disk material.
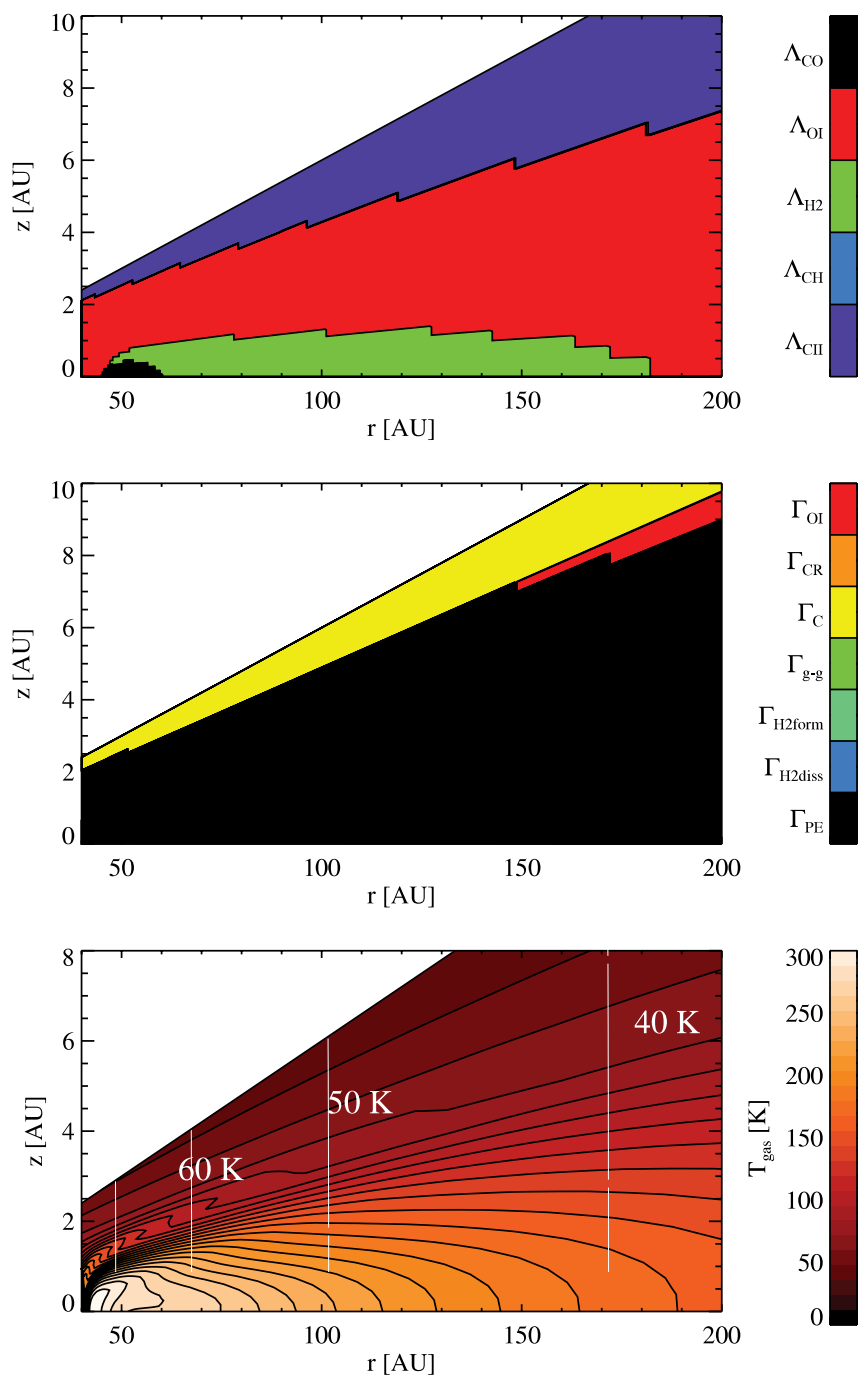

FIG. 5.- Two-dimensional gas temperatures in the best-fit disk model ( $M_{\text {disk }}=13 M_{\oplus}, \epsilon=2.5, h=0.02$. Shown are the most important heating (top) and cooling (middle) processes, as well as the gas temperature (bottom). The dust temperature, which depends only on radius, is overlaid in white contour lines (steps of $10 \mathrm{~K}$ ).

The densities in the disk are too low for efficient gas-dust coupling and thus the gas finds its own equilibrium temperature determined mainly by photoelectric heating and line cooling. The most important cooling lines from surface to midplane are [C II], [O I], and $\mathrm{H}_{2}$. $\mathrm{CO}$ abundances are only high in a region between 45 and $70 \mathrm{AU}$ (Fig. 3). Outside that region, CO cooling is less important for the energy balance. Figure 5 summarizes the most important heating and cooling processes and also shows the resulting gas temperature structure. The disk surface stays well below $100 \mathrm{~K}$ due to efficient fine-structure line cooling. The molecular cooling is, however, less efficient in competing with the photoelectric heating from the large silicate grains (Kamp \& van Zadelhoff 2001), leading to temperatures of a few $100 \mathrm{~K}$ in the disk interior.

In order to test the robustness of the best-fit model to the gas properties, we used this model to predict the CO $J=3-2$ spectrum. It compares favorably with the spectrum observed by Dent et al. (2005) reproduced in Figure 6. The thick solid line shows the $J=3-2$ spectrum predicted from the best-fit disk model, while the thin solid line shows the observed JCMT spectrum.

Although the observed spectrum is noisy and likely subject to an absolute calibration uncertainty, the overall agreement is within 


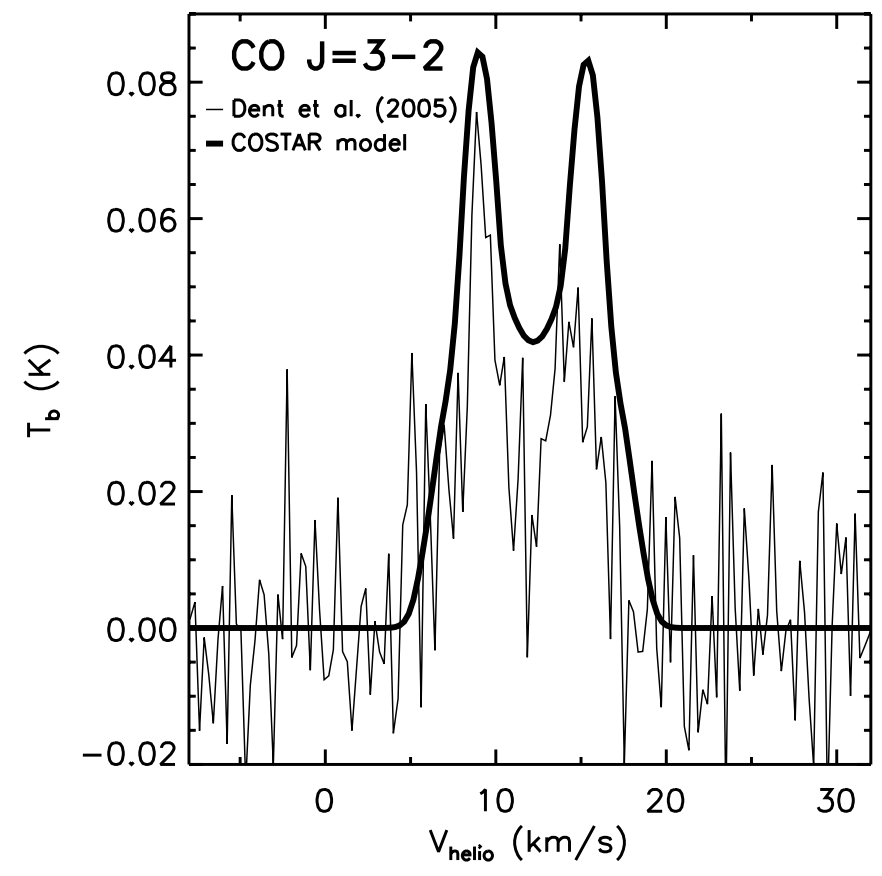

FIG. 6. $-\mathrm{CO} J=3-2$ spectrum predicted for the model that provides the best fit to the resolved $J=2-1$ emission (thick solid line), compared with the Dent et al. (2005) JCMT CO $J=3-2$ spectrum (thin solid line). The $x$-axis shows heliocentric velocity while the $y$-axis gives the JCMT main beam brightness temperature.

$\sim 30 \%$, which is very good given that the CO $J=3-2$ spectrum was not used a priori to determine these model parameters.

\section{DISCUSSION}

The processes determining the amount and distribution of gas and dust in transition disks like that around 49 Ceti are the same processes that shape the features of emergent planetary systems around these young stars. Resolved observations of individual disks in this phase are desirable to address such basic questions as when in the lifetime of a star its disk disperses, whether the gas clears before the dust, and whether the disk clears from the center or in a radially invariant manner.

In the 49 Ceti system, the infrared dust properties appear similar to those of a debris disk (Wahhaj et al. 2007). Yet, observations presented here indicate that a substantial quantity of molecular gas persists in the outer disk, between radii of 40 and $200 \mathrm{AU}$, where photochemistry from stellar and interstellar radiation dominates. The lack of molecular gas emission inside this radius as indicated by our observations, combined with the lack of dust emission within a radius of $30 \mathrm{AU}$ inferred by Wahhaj et al. (2007), implies that the 49 Ceti system appears to be clearing its gas and dust from the center out. The mechanism responsible for this central clearing is not indicated; in general, the best-developed theories to explain this transitional morphology are (1) central clearing through the influence of a massive planet and (2) photoevaporation by radiation from the central star.

The clearing of gaps and inner holes has long been predicted as a consequence of the formation of massive planets in circumstellar disks (e.g., Lin \& Papaloizou 1986; Bryden et al. 1999). In the case of 49 Ceti, the formation of a Jupiter-mass planet would be required at a distance of $\sim 40 \mathrm{AU}$ from the star, roughly the inner radius of the observed hole in the gas distribution. Such a scenario could also help to explain the size segregation of dust grains observed by Wahhaj et al. (2007); a predicted conse- quence of inner disk clearing by gravitational influence of a massive planet is a filtration of dust grains by size, with only those below a certain threshold (typically 1-10 $\mu \mathrm{m}$ ) accreted across the gap along with a reduced amount of gas (Rice et al. 2006). However, this scenario ultimately requires the accretion of substantial amounts of gas into the inner disk, and searches for molecular gas in the inner disk of 49 Ceti (Chen et al. 2006; Carmona et al. 2008) have not detected such a population. Another indication that an inner hole is likely caused by a massive planet in formation would be nonaxisymmetric features resulting from its gravitational influence, such as spiral waves. While the CO emission from 49 Ceti does not appear asymmetric within the limits of the SMA observations, more sensitive spatially resolved observations could address this hypothesis.

The absence of gas in the inner disk is, however, consistent with a photoevaporation scenario: as the photoevaporative wind produced by stellar radiation becomes comparable to the accretion rate in the disk, material within the gravitational radius $R_{g}=G M_{\star} / c_{s}^{2}$ will quickly drain onto the star, leaving an evacuated inner hole free of gas and dust (e.g., Hollenbach et al. 1994; Alexander et al. 2006). The gravitational radius for 49 Ceti is roughly $20 \mathrm{AU}$, which is comparable to the inferred inner radius of $40 \mathrm{AU}$ for the outer disk. The larger outer radius may in fact be consistent with the later stages of photoevaporation, after the inner disk has become optically thin to ultraviolet radiation and the inner disk radius slowly increases under the influence of the photoevaporative wind (Alexander et al. 2006). Alexander \& Armitage (2007) propose a method of discriminating between inner holes caused by photoevaporation and those caused by the formation of a giant planet, involving a simple comparison between two observables: the disk mass and the accretion rate. As there is no measured accretion rate for 49 Ceti, we cannot apply the criteria presented by these authors; however, we note that the low disk mass does indeed fall within the parameter space consistent with a photoevaporative scenario. Further observations are necessary to determine the origin of the inner hole; in particular, stringent limits on the accretion rate could suggest a photoevaporative mechanism.

There are few disks that appear to be in a similar evolutionary stage to that of 49 Ceti; a rare example is the disk around the A star HD 141569. Like 49 Ceti, it hosts a disk composed primarily of submicron-size grains with infrared properties approaching those of a debris disk (Wahhaj et al. 2007; Marsh et al. 2002) while still retaining a substantial quantity of molecular gas with central region clear of gas emission, in this case out to a radius of $\sim 11$ AU (Goto et al. 2006; Brittain et al. 2007). It exhibits a transitional SED (Merín et al. 2004), and observations of the rovibrational $\mathrm{CO}$ spectrum reveal gas with disparate rotational and vibrational temperatures (200 and $5000 \mathrm{~K}$, respectively; Brittain et al. 2007), indicative of UV fluorescence on the outer edges of an inner disk region cleared of gas and dust. An analysis of the chemistry and gas properties of the system similar to the one presented here for 49 Ceti was conducted by Jonkheid et al. (2006). While the presence and extent of the inner hole are clearly indicated, the physical origin of this clearing is less obvious. The $\mathrm{Br} \gamma$ profile is indicative of substantial accretion, and Brittain et al. (2007) deem a photoevaporative clearing mechanism unlikely, due to the large column density outside the cleared region and the lack of evidence for a photoevaporative wind in the farultraviolet (Martin-Zaïdi et al. 2005). However, Merín et al. (2004) place a much lower limit of $10^{-11} M_{\odot} \mathrm{yr}^{-1}$ on the accretion rate, based on the assumed gas : dust ratio of 100 and the low optical depth of the inner disk, which would be much more consistent with a photoevaporation scenario. Goto et al. (2006) note that the rough 
coincidence of the inner rim of the disk with the gravitational radius suggests that photoevaporation in concert with viscous accretion is a likely cause for the inner disk clearing.

Whatever the origin of their morphology, the observed gas and dust properties indicate that the disks surrounding both 49 Ceti and HD 141569 appear to be in a transitional state of evolution during which the dust properties are beginning to appear more like those of a debris disk, while the gas is in the process of being cleared from the disk from the center out.

\section{CONCLUSIONS}

The SMA CO $J=2-1$ observations presented here provide the first spatially resolved observations of molecular gas in the 49 Ceti system. The data reveal a surprisingly extended and complex molecular gas distribution in rotation about the central star, viewed approximately edge-on and clear of molecular gas emission in the central region of the disk. Modeling the disk structure and chemistry in this system indicates that the in- ner disk is entirely devoid of molecular gas due to irradiation by the central star, while a ring of molecular gas persists between 40 and $200 \mathrm{AU}$, subject to photodissociation at the inner edge by stellar radiation. The disk model presented here reproduces well the observed properties of the system, including the resolved $\mathrm{CO}$ $J=2-1$ emission, the CO $J=3-2$ spectrum, and the spectral energy distribution. With dust properties similar to those of a debris disk and a substantial reservoir of gas maintained in the outer disk, 49 Ceti appears to be a rare example of a system in a late stage of transition between a gas-rich protoplanetary disk and a tenuous, gas-free debris disk.

The authors would like to thank Bill Dent for providing the JCMT CO $J=3-2$ spectrum. Partial support for this work was provided by NASA Origins of Solar Systems Program grant NAG5 11777. A. M. H. acknowledges support from a National Science Foundation Graduate Research Fellowship.
Alexander, R. D., \& Armitage, P. J. 2007, MNRAS, 375, 500

Alexander, R. D., Clarke, C. J., \& Pringle, J. E. 2006, MNRAS, 369, 229

Aumann, H. H., et al. 1984, ApJ, 278, L23

Beckwith, S. V. W., \& Sargent, A. I. 1991, ApJ, 381, 250

Bockelée-Morvan, D., André, P., Colom, P., Colas, F., Crovisier, J., Despois, D., \& Jorda, L. 1994, in Circumstellar Dust Disks and Planet Formation, ed. R. Ferlet \& A. Vidal-Madjar (Paris: Editions Frontières), 341

Brittain, S. D., Simon, T., Najita, J. R., \& Rettig, T. W. 2007, ApJ, 659, 685

Bryden, G., Chen, X., Lin, D. N. C., Nelson, R. P., \& Papaloizou, J. C. B. 1999 , ApJ, 514, 344

Cardelli, J. A., Clayton, G. C., \& Mathis, J. S. 1989, ApJ, 345, 245

Carmona, A., van den Ancker, M. E., Henning, T., Goto, M., Fedele, D., \& Stecklum, B. 2008, A\&A, 478, 795

Chen, C. H., et al. 2006, ApJS, 166, 351

Dent, W. R. F., Greaves, J. S., \& Coulson, I. M. 2005, MNRAS, 359, 663

Goto, M., Usuda, T., Dullemond, C. P., Henning, T., Linz, H., Stecklum, B., \& Suto, H. 2006, ApJ, 652, 758

Habing, H. J. 1968, Bull. Astron. Inst. Netherlands, 19, 421

Hoffleit, D., \& Jaschek, C., eds. 1991, The Bright Star Catalogue (New Haven: Yale University Observatory)

Hogerheijde, M. R., \& van der Tak, F. F. S. 2000, A\&A, 362, 697

Holland, W. S., et al. 1998, Nature, 392, 788

Hollenbach, D., Johnstone, D., Lizano, S., \& Shu, F. 1994, ApJ, 428, 654

Jonkheid, B., Kamp, I., Augereau, J.-C., \& van Dishoeck, E. F. 2006, A\&A, 453, 163

Jura, M., Malkan, M., White, R., Telesco, C., Pina, R., \& Fisher, R. S. 1998, ApJ, 505, 897

Jura, M., Zuckerman, B., Becklin, E. E., \& Smith, R. C. 1993, ApJ, 418, L37

\section{REFERENCES}

Kamp, I., \& Bertoldi, F. 2000, A\&A, 353, 276

Kamp, I., \& van Zadelhoff, G.-J. 2001, A\&A, 373, 641

Lin, D. N. C., \& Papaloizou, J. 1986, ApJ, 309, 846

Mamajek, E. E. 2005, ApJ, 634, 1385

Marsh, K. A., Silverstone, M. D., Becklin, E. E., Koerner, D. W., Werner, M. W., Weinberger, A. J., \& Ressler, M. E. 2002, ApJ, 573, 425

Martin-Zaïdi, C., Deleuil, M., Simon, T., Bouret, J.-C., Roberge, A., Feldman, P. D., Lecavelier Des Etangs, A., \& Vidal-Madjar, A. 2005, A\&A, 440, 921 Merín, B., et al. 2004, A\&A, 419, 301

Ortega, V. G., et al. 2002, ApJ, 575, L75

Rice, W. K. M., Armitage, P. J., Wood, K., \& Lodato, G. 2006, MNRAS, 373, 1619

Sadakane, K., \& Nishida, M. 1986, PASP, 98, 685

Siess, L., Dufour, E., \& Forestini, M. 2000, A\&A, 358, 593

Song, I., Sandell, G., \& Friberg, P. 2004, in ASP Conf. Ser. 324, Debris Disks and the Formation of Planets, ed. L. Caroff et al. (San Francisco: ASP), 250 Stauffer, J. R., Hartmann, L. W., \& Barrado y Navascues, D. 1995, ApJ, 454, 910 Sylvester, R. J., Skinner, C. J., Barlow, M. J., \& Mannings, V. 1996, MNRAS, 279,915

Thi, W. F., et al. 2001a, Nature, 409, 60

2001b, ApJ, 561, 1074

Wahhaj, Z., Koerner, D. W., \& Sargent, A. I. 2007, ApJ, 661, 368

Weinberger, A. J., Becklin, E. E., Schneider, G., Smith, B. A., Lowrance, P. J., Silverstone, M. D., Zuckerman, B., \& Terrile, R. J. 1999, ApJ, 525, L53 Zuckerman, B. 2001, ARA\&A, 39, 549

Zuckerman, B., Forveille, T., \& Kastner, J. H. 1995, Nature, 373, 494

Zuckerman, B., et al. 2001, ApJ, 562, L87 